\title{
ABSTRACT CLASS FORMATIONS
}

\author{
BY K. GRANT AND G. WHAPLES
}

Communicated by Alex Rosenberg, February 16, 1961

Let $k_{0}$ be any field, $\Omega$ a normal separable extension of it, and $R=R\left(k_{0}, \Omega\right)$ the set of all fields of finite degree over $k_{0}$ which are contained in $\Omega$. A class formation is a family of groups $\{E(K)\}_{K \in R}$, indexed by $R$, together with a family of monomorphisms $\Phi_{K, L}: E(K) \rightarrow E(L)$, defined for $K \subset L$, satisfying the Artin-TateKawada axioms [1]. These axioms state that every automorphism of a $K \in R$ which is identity on $k_{0}$ defines an automorphism of $E(K)$; that these automorphisms commute in a reasonable way with the $\Phi_{K, L}$; that if $k, K \in R$ and $k$ is fixed point field for a finite group $H$ of automorphisms of $K$ then

$$
(E(K))^{H}=\Phi_{k, K} E(k),
$$

$(E(K))^{H}$ denoting the subgroup of $E(K)$ on which $H$ acts simply; and that

$$
H^{1}(G, E(K)),=0 ; \quad H^{2}(G, E(K)) \text { cyclic of order \#G }
$$

for all $K \in R$, all finite groups $G$ of automorphisms of $K / k_{0}$, where \#G denotes the number of elements of $G$. From well-known results of J. Tate [2] it follows that (2) is equivalent to

$$
H^{r}(G, E(K)) \cong H^{r-2}(G, Z) \quad \text { for all } r,
$$

where $Z$ denotes the additive group of ordinary integers with $G$ acting simply on it.

The best known examples of class formations are furnished by local and global class field theory but Kawada has found several others. In all these examples the groups $E(K)$ are defined by use of the structure of the fields $K$, so that by use of the class formation one can deduce connections between the arithmetic of the fields $K \in R$ and the algebraic extensions of these fields.

In this note we entirely ignore the structure of the fields $K \in R$, asking merely whether, for given $R$, there exists any family of groups indexed by $R$ which form a class formation. Thus we use the fields in $R$ merely as indices. Indeed, since the fields in $R$ are in one-one correspondence with the open subgroups of the topological Galois group $G_{\infty}$ of $\Omega / k_{0}$ we could formulate our question in the following more general way: Given a topological group $G_{\infty}$ which is projective limit of a directed system of finite groups does there exist a family of groups, indexed by the set of all open subgroups of $G_{\infty}$, which 
satisfies the axioms of Artin-Tate-Kawada when these axioms are stated in terms of the Galois groups $G(\Omega / K)$ rather than in terms of the fields $K$ ? In this note we use the language of fields, but only for convenience; our results can easily be translated into the language of groups, and remain true. We shall prove that for every $\Omega / k_{0}$, or for every $G_{\infty}$, as described above, there always exists an (abstract) class formation. Of course this is of far less interest than the study of class formations with an arithmetical meaning; but it does add to our understanding of the nature of class formations, and it proves that the existence of a class formation for $\Omega / k_{0}$ imposes no condition whatever on the Galois group of $\Omega / k_{0}$.

There are, no doubt, many constructions for abstract class formations, some even simpler than the one we are going to give. We are still investigating this. From $\left(2^{\prime}\right)$ it is clear that we can do it if we can find a family of $E(K)$ which shift the dimension by 2 and permit a system of monomorphisms $\Phi_{K, L}$.

If $G$ is any finite group let $Z[G]$ denote its group ring over $Z$ and $X(G)=Z[G] \otimes_{Z} Z[G]$, defined as $G$-module by: $\rho \sum n_{\sigma, \tau} \sigma \otimes r$ $=\sum n_{\sigma, \tau} \rho \sigma \otimes \rho \tau$. Let $\lambda$ be the homomorphism $Z[G] \rightarrow Z$ defined by $\sum n_{\sigma} \sigma \rightarrow \sum n_{\sigma}$. Let $N(G)$ be the kernel of $\lambda$ and $\Pi(G)=N(G) \otimes_{z} N(G)$. Let $R$ be as above and for every $K \in R$ let $R(K)$ denote the set of all fields $N \in R$ which contain $K$ and are normal over $k_{0}$. If $N$ is such a field define $G_{N}=G\left(N / k_{0}\right)$ and $H_{N}=G(N / K)$, and define $M(K, N)$ to be $\Pi\left(G_{N}\right)^{H_{N}}$. If $N^{\prime} \subset N$ with $N^{\prime}, N \in R(K)$ define $\Psi_{N^{\prime}, N}: X\left(G_{N^{\prime}}\right)$ $\rightarrow X\left(G_{N}\right)$ by: $\sum_{\sigma^{\prime}, \tau^{\prime} \in G_{N^{\prime}}} n_{\sigma^{\prime}, \tau^{\prime}} \sigma^{\prime} \otimes \tau^{\prime} \rightarrow \sum_{\sigma, \tau \in G_{N}} n_{\phi(\sigma), \phi(\tau)} \sigma \otimes \tau$ where $\phi$ is the natural homomorphism (restriction to $N^{\prime}$ ) of $G_{N}$ onto $G_{N^{\prime}}$. One can prove that the mappings $\Psi_{N^{\prime}, N}$ enjoy reasonable transitivity and commutativity properties, and that $\Psi_{N^{\prime}, N}$ induces a monomorphism $\eta_{N^{\prime}, N}^{K}$ of $M\left(K, N^{\prime}\right)$ into $M(K, N)$. For each $K \in R$ we have a directed system of groups $\left\{M(K, N), \eta_{N^{\prime}, N}^{K}\right\}_{N \in R(k)}$. Define $E(K)=$ direct limit $_{R(K)} M(K, N)$.

If $k \subset K$, both being in $R$, then $R(K)$ is a cofinal subset of $R(k)$. Also if $k \subset K$ and $N$ is in both $R(k)$ and $R(K)$ then $M(k, N) \subset M(K, N)$. Let $i_{\boldsymbol{k}, K}^{N}: M(k, N) \rightarrow M(K, N)$ denote the inclusion map and $\Phi_{k, K}$ the direct limit, over $N \in R(K)$, of the $i_{k, K}^{N}$; then $\Phi_{k, K}$ is a monomorphism of $E(k)$ into $E(K)$ and the family of mappings $\Phi_{k, K}$ has the transitivity and commutativity properties required for a class formation; that is, $\left\{E(K), \Phi_{k, K}\right\}$ is a "formation" in the sense of Kawada [1].

It remains to prove (2). For any finite group $G$ we have exact sequences

$$
\begin{aligned}
& 0 \rightarrow N(G) \rightarrow Z[G] \rightarrow Z \rightarrow 0, \\
& 0 \rightarrow \Pi(G) \rightarrow Z[G] \otimes_{Z} N(G) \rightarrow N(G) \rightarrow 0 .
\end{aligned}
$$


If $H$ is any subgroup of $G$ then these are all $H$-modules and $Z[G]$ and $Z[G] \otimes_{Z} N(G)$ split over $H$. So from (3) we get $H^{r}(H, N(G))$ $\cong H^{r-1}(H, Z)$ and from (4) we get $H^{r}(H, \Pi(G)) \cong H^{r-1}(H, N(G))$ ordinary dimension shift-hence

$$
H^{r}(H, \Pi(G)) \cong H^{r-2}(H, Z)
$$

for all $r$ and all subgroups $H$ of $G$.

Now let $k_{0} \subset k^{\prime} \subset k \subset N$ with $k, k^{\prime} \in R$ and $N \in R(k)$. Let $G=G\left(N / k_{0}\right)$, $H=G(N / k)$, and $H^{\prime}=G\left(N / k^{\prime}\right)$. Suppose that $k / k^{\prime}$ is normal and let $F=H^{\prime} / H=G\left(k / k^{\prime}\right)$. We have to prove that

$$
H^{1}(F, E(k))=(0) ; \quad H^{2}(F, E(k)) \cong Z /(\# F) Z .
$$

From our definitions and the usual theory of cohomology groups of direct limits, (6) will follow if we can prove it with $E(k)$ replaced by $M(k, N)$. Now the sequence,

$$
0 \rightarrow H^{1}\left(F, \Pi(G)^{H}\right) \rightarrow H^{1}\left(H^{\prime}, \Pi(G)\right) \rightarrow H^{1}(H, \Pi(G))
$$

is exact, where the first mapping is lift and the second restriction. From (5) we see that $H^{1}\left(H^{\prime}, \Pi(G)\right) \cong H^{1}(H, \Pi(G)) \cong H^{-1}(H, Z)=(0)$. So (7) gives $H^{1}\left(F, \Pi(G)^{H}\right) \cong H^{1}\left(H^{\prime}, \Pi(G)\right) \cong(0)$ or, by definition of $M(k, N)$ :

$$
H^{1}(F, M(k, N))=(0) \quad \text { for all } k, N .
$$

This being so the sequence

$$
0 \rightarrow H^{2}(F, M(k, N)) \rightarrow H^{2}\left(H^{\prime}, \Pi(G)\right) \rightarrow H^{2}(H, \Pi(G))
$$

is exact. From (5), the latter two groups are cyclic of orders $\# H^{\prime}$ and $\# H$ respectively. So from (9) we see that $H^{2}(F, M(k, N))$ is cyclic of order divisible by $\# H^{\prime} / \# H=\# F$. On the other hand, if $\# F=n$, then the composite mapping

$$
H^{2}\left(H^{\prime}, \Pi(G)\right) \stackrel{\text { rst }}{\longrightarrow} H^{2}(H, \Pi(G)) \stackrel{\operatorname{tr}}{\longrightarrow} H^{2}\left(H^{\prime}, \Pi(G)\right)
$$

is the $n$th power mapping on $H^{2}\left(H^{\prime}, \Pi(G)\right)$, so the kernel of the restriction map in (10) (which is isomorphic to $H^{2}(F, M(k, N))$ by (9)) is contained in the kernel of the $n$th power mapping of a cyclic group. So $H^{2}(F, M(k, N))$ is cyclic of order dividing $n$, and this completes our proof.

\section{REFERENCES}

1. Y. Kawada, Class formations, Duke Math. J. vol. 22 (1955) pp. 165-177.

2. J. Tate, The higher dimensional cohomology groups of class field theory, Ann. of Math. vol. 56 (1952) pp. 294-297. 